\title{
Influencia de texturas superficiales acondicionadas en el desempeño de cojinetes planos operando en condiciones HL
}

\section{Influence of Conditioned Surface Textures on Plain Journal Bearing Performance Working on HL Conditions}

\author{
Suárez-Bustamante F.A. \\ Ciencia y Tecnología de Materiales (CTM) \\ Grupo de Tribología y Superficies (GTS) \\ Universidad Nacional de Colombia, Sede Medellín \\ E-mail:alexandersb@gmail.com \\ Toro-Botero F.M. \\ Universidad Nacional de Colombia, Sede Medellin \\ Posgrado en Aprovechamiento de Recursos Hidráulicos \\ E-mail:fmtoro@unalmed.edu.co
}

\author{
Vélez-Restrepo J.M. \\ Ciencia y Tecnología de Materiales (CTM) \\ Grupo de Tribología y Superficies (GTS) \\ Universidad Nacional de Colombia, Sede Medellin \\ E-mail:jmvelez@unalmed.edu.co
}

Información del artículo: agosto de 2010, reevaluado: mayo de 2011, aceptado: septiembre de 2011

\section{Resumen}

Como respuesta a la necesidad de disminuir las pérdidas por fricción generadas en cojinetes, sellos dinámicos, anillos de pistones y herramientas de corte o de conformado plástico, entre otros, se ha abordado el estudio de sistemas mecánicos, cuyos elementos tienen superficies con texturas acondicionadas. Modelos teóricos y resultados experimentales han mostrado que es posible obtener un mejor desempeño tribológico en estos sistemas, con respecto a los no texturizados operando en las mismas condiciones. Este trabajo tiene como objetivo presentar un modelo numérico para establecer relaciones entre las condiciones de operación (carga, velocidad y viscosidad dinámica), el espesor mínimo de película lubricante, el coeficiente de fricción y la micro-topografía acondicionada de un cojinete plano de deslizamiento operando en régimen hidrodinámico (HL). Además, a diferencia de otros trabajos similares, el modelo construido permite estudiar el efecto combinado de las micro-cuñas que operan como micro-cojinetes y la macrocuña convergente típica de estos elementos de estudio.

\section{Descriptores}

- tribología

- lubricación

- superficies texturizadas

- cojinetes

- fricción

- hidrodinámica 


\begin{abstract}
As an answer to the need for reducing friction losses on bearings, dynamic seals, piston rings, cutting tools and others, a lot of work has been dedicated to mechanical systems study whose surfaces have been textured in a controlled way. Theoretical models and experimental results have shown improvements on the tribological performance of these systems regarding untextured systems, working under the same conditions. This paper presents a numerical model for getting relationships among the operational conditions (load, speed, and dynamic viscosity), the minimum lubricate film thickness, the friction coefficient and the conditioned micro-topography of a plain sliding bearing working under a hydrodynamic regime. Moreover, regarding other similar works the constructed model allows studying the combined effect of the micro-wedges that work as micro-bearings, and the typical convergent macro-wedge of these study elements.
\end{abstract}

\section{Introducción}

Según Ronen et al. (2001), cerca de 40\% de la energía total transformada por un motor de combustión interna de un automóvil se disipa como trabajo de las fuerzas de fricción. La disminución de las pérdidas por fricción es un factor clave para lograr un mejor uso de los combustibles y la protección del medio ambiente. El sistema anillo-pistón-camisa, responde por pérdidas equivalentes a 50 o $60 \%$ del total de las pérdidas por fricción en un motor de estos. Se justifica así, la importancia económica y ambiental de realizar investigaciones tendientes a mejorar el desempeño de este sistema en los motores de combustión interna (Kligerman y Etsion, 2002).

Esto ha generado una tendencia hacia el diseño y fabricación de elementos mecánicos más eficientes y con una mayor relación de potencia manejada por unidad de volumen. Para alcanzar esto, los esfuerzos de los investigadores se han centrado en las siguientes áreas (Edemir, 2005):

a) Desarrollo de lubricantes de menor viscosidad y mayor duración.

b) Desarrollo de materiales de baja fricción y de recubrimientos duros.

c) Diseño y fabricación de superficies óptimas de ingeniería mediante el acondicionamiento de sus microtopografías y/o texturas superficiales.

Esta última área busca obtener micro-topografías que permitan minimizar las fuerzas de fricción involucradas durante la operación de elementos mecánicos de uso común, como son: sellos dinámicos, cojinetes de empuje, rodamientos y sistemas anillos del pistón-ca- misa del pistón, entre otros (Ronen et al., 2001), (Anno et al., 1968) y (Ryk et al., 2002).

Este artículo tiene como objetivo principal presentar un modelo numérico que permite establecer relaciones entre las condiciones de operación (carga, velocidad y viscosidad dinámica), el espesor mínimo de película lubricante, el coeficiente de fricción y la micro-topografía acondicionada de un cojinete plano de deslizamiento operando en régimen hidrodinámico (HL). A diferencia de otros trabajos similares encontrados en la literatura (Ronen et al., 2001; Anno et al., 1968) , el modelo construido permite estudiar el efecto combinado de las micro-cuñas que operan como micro-cojinetes, y la macro-cuña convergente típica de estos elementos de estudio. Otro elemento diferenciador de este trabajo está asociado con la geometría de la textura acondicionada que se estudia, un patronado constituido por surcos trapezoidales orientados perpendicularmente respecto a la dirección de deslizamiento. Este tipo de patronado puede utilizarse convenientemente para hacer aproximaciones de otras texturas con geometrías más complejas, como por ejemplo, un perfil sinusoidal; u otras más básicas como una textura tipo diente de sierra. Este último aspecto le confiere al sistema de estudio una alta flexibilidad y adaptabilidad al momento de ser empleado en simulaciones.

\section{Lubricación hidrodinámica y texturizado de su- perficies}

Las condiciones de operación (carga, velocidad y temperatura) determinan la estabilidad y el espesor de las películas lubricantes (Hutchings, 1992). Para presiones de contacto altas o las velocidades de deslizamiento bajas, las fuerzas hidrodinámicas no alcanzan a mantener 
un espesor de película lubricante capaz de separar completamente las asperezas de las superficies y evitar las interacciones o colisiones entre éstas. Como resultado, se generan altas fuerzas de fricción y elevadas tasas de desgaste. Además, la macro-geometría de las superficies es otro elemento a considerar, ya que condiciona el tipo de contacto que existe entre las superficies, el cual puede clasificarse como conforme (no localizado) o no conforme (localizado).

El trabajo de Osborne Reynolds (1880) es aún la base de la teoría matemática para estudiar el fenómeno de lubricación hidrodinámica (Ronen et al., 2001 y Schlichting, 1979), su gran aporte se ve plasmado en la siguiente ecuación,

$\frac{\partial}{\partial x}\left(h^{3} \frac{\partial p}{\partial x}\right)+\frac{\partial}{\partial y}\left(h^{3} \frac{\partial p}{\partial y}\right)=6 \mu U \frac{\partial h}{\partial x}+12 \mu \frac{\partial h}{\partial t}$

donde

hy $p$ son el espesor y la presión de la película lubricante en el punto de coordenadas $(x, y)$ respectivamente,

$\mu \quad$ la viscosidad dinámica del lubricante,

$U$ la velocidad relativa entre las superficies y

$t$ el tiempo.

Para el caso de un cojinete de deslizamiento, se emplea el parámetro de lubricación $(H=\mu U / P)$, donde $P$ es la carga normal) para analizar los cambios del coeficiente de fricción en función de las condiciones de operación. La figura 1 esquematiza esta relación; la curva mostrada se denomina curva de Stribeck (Hutchings, 1992) o de McKee (Bouden y Tabor, 2001). En ella se observan tres regiones con comportamientos diferenciados del coeficiente de fricción, de la estabilidad de la película lubricante y de su espesor, definiendo cada una de éstas un régimen de lubricación característico.

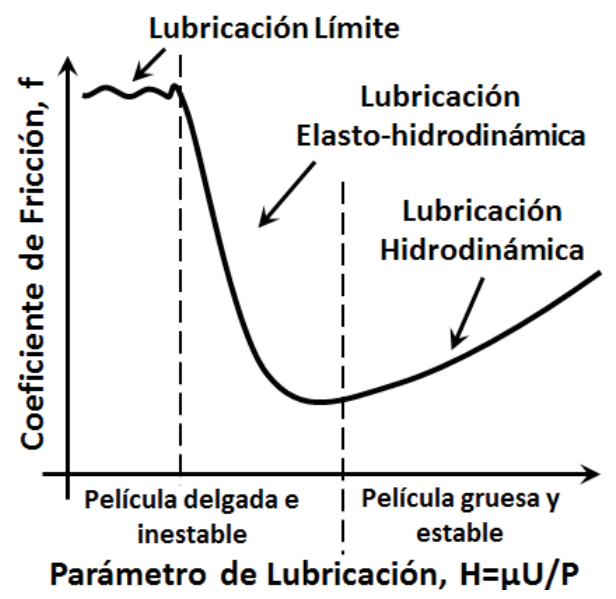

Figura 1. Curva de Stribeck
Las asperezas que constituyen la rugosidad pueden ser del mismo orden de magnitud del espesor de la película lubricante, por lo tanto, su influencia en la estabilidad de la película y en la posibilidad de alcanzar un régimen de lubricación hidrodinámico es importante. Se han propuesto varios modelos de lubricación hidrodinámica para cojinetes con superficies rugosas; de estos se destacan los modelos de Christersen y Tonder por su naturaleza estocástica (Christensen, 1969; Christensen y Tonder, 1971). Actualmente, se realizan estudios teóricos tendientes a determinar los efectos de la rugosidad superficial en las características dinámicas de cojinetes deslizantes (Ronen et al., 2001; Kligerman y Etsion, 2002; Letalleur et al., 2001; Wang y Zhu, 2005; Ryk et al., 2002; Etsion et al., 2004; Kovalchenko et al., 2005; Chiang et al., 2005; Suárez, 2008). Este aspecto ha sido uno de los elementos que han motivado el desarrollo del trabajo que se presenta en este artículo.

El trabajo de Anno et al. (1968), sobre lubricación de superficies con texturas acondicionadas, explica el fenómeno de lubricación hidrodinámica en sistemas rotativos de caras paralelas, nominalmente planas, como son los cojinetes de empuje y los sellos dinámicos. La presencia de superficies planas e inclinadas en las cimas de las asperezas creadas para sus experimentos, permitieron observar cómo el efecto combinado de pequeñas cuñas hidrodinámicas es capaz de generar una película estable con el espesor necesario para soportar la carga. Este hecho ha motivado a un gran número de investigadores a profundizar en este tema, debido a las posibilidades de reducir la fricción y el desgaste en cojinetes; además de permitir que los sistemas puedan trabajar en condiciones más extremas (mayores cargas normales y/o menores velocidades de deslizamiento) sin salirse de su régimen hidrodinámico. El trabajo realizado por los profesores Etsion y Kligerman en conjunto con sus colaboradores (Ronen et al., 2001; Kligerman y Etsion, 2002; Ryk et al., 2002; Etsion et al., 2004; Kovalchenco et al., 2005) es un claro ejemplo que evidencia los beneficios que pueden obtenerse mediante el diseño adecuado de la micro-topografía de las superficies de elementos que deslizan entre sí. Ellos han trabajado en la exploración teórico-experimental del uso del texturizado de superficies con láser (LST) en sistemas de anillos y camisas del pistón de motores de combustión interna. Las simplificaciones realizadas en sus modelos teóricos y de laboratorio pueden aplicarse a un gran número de elementos mecánicos, ya que se basan en la operación de cojinetes planos de deslizamiento (Ronen et al., 2001). Como este grupo de trabajo existen otros que actualmente investigan sobre los efectos de las texturas superficiales acondicionadas en los fenómenos de fric- 
ción y desgaste de diversos tribo-sistemas empleando distintas aproximaciones (teóricas y experimentales) y motivados por diversos intereses particulares (Pettersson y Jacobson, 2003; Pettersson, 2005; Tonder, 2004; Wang y Zhu, 2005; Wang et al., 2003 y Wang et al., 2006).

\section{El modelo elaborado}

Para establecer las relaciones entre la microtopografía superficial, las condiciones de operación (definidas éstas por las variables carga, velocidad y viscosidad dinámica) y el coeficiente de fricción en un cojinete plano de deslizamiento operando en un régimen $\mathrm{HL}$, fue necesario construir un modelo que combinara los efectos de dos escalas geométricas relacionadas con las diversas cuñas formadas en estos tribo-sistemas. Una de estas escalas, la macro-escala, es representada por la cuña formada por la superficie nominalmente plana del cojinete y el contra-cuerpo; y la otra escala, denominada en este trabajo como la micro-escala, es aquella representada por las pequeñas cuñas convergentes y divergentes asociadas a las asperezas creadas convenientemente (o si se prefiere, a la textura acondicionada como tal). A continuación, se muestran los aspectos más relevantes relacionados con el modelo propuesto.

\section{El sistema de estudio}

El sistema modelado (figura 2a) consta de un cuerpo con una superficie nominalmente plana y libre de asperezas en movimiento relativo, con respecto a otro cuerpo cuya superficie plana ha sido texturizada; las superficies se encuentran separadas entre sí por un lubricante y forman una macro-cuña convergente carac- terizada por el ángulo $\theta$. La textura seleccionada está compuesta por surcos trapezoidales orientados en dirección perpendicular a la velocidad de deslizamiento (figura 2b); en ella se desprecian los efectos de rugosidades más pequeños que las inducidas en el elemento texturizado. Adicionalmente, se considera que el sistema se encuentra en un estado estable.

\section{El mecanismo}

Con base en los trabajos previos de Anno et al. (1968) y Ronen et al. (2001), se identificó el mecanismo básico que explica los cambios en el desempeño tribológico de este sistema, con respecto a un cojinete no texturizado. Las irregularidades superficiales crean microcuñas convergentes y divergentes que alteran el flujo del lubricante entre las superficies de los cuerpos en movimiento relativo, generando a su vez, cambios en las distribuciones de presiones sobre las superficies lubricadas.

\section{Consideraciones básicas del modelo de Reynolds para $\mathrm{HL}$}

Partiendo del modelo de Reynolds para un cojinete plano de longitud infinita, en el cual las superficies de los cuerpos son totalmente planas, se hizo una generalización para considerar el efecto del texturizado. El modelo de Reynolds supone constante la viscosidad dinámica del fluido incompresible utilizado como lubricante y desprecia los efectos asociados a su calentamiento, producto de la disipación viscosa. Así, los efectos de las variables térmicas y mecánicas se consideran independientes. Sin embargo, como primera aproximación al a) Cojinete plano de deslizamiento

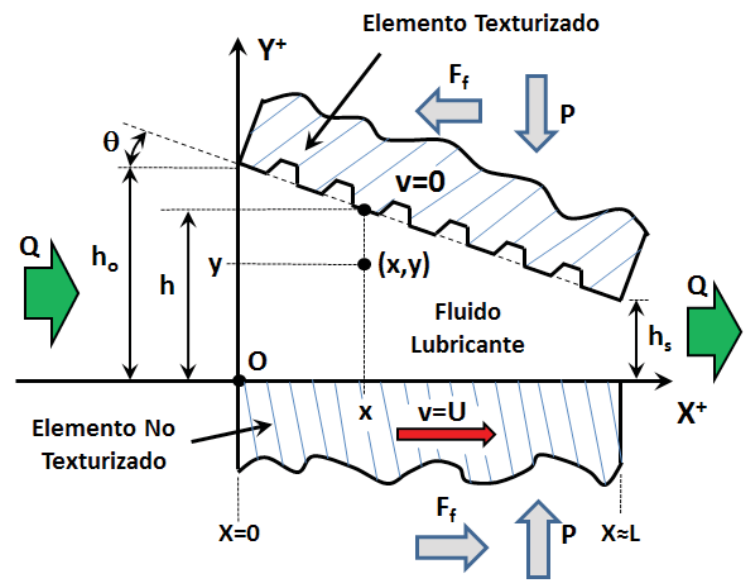

b) Geometría del elemento texturizado de longitud $L$ y $\operatorname{con} n \operatorname{surcos}$.

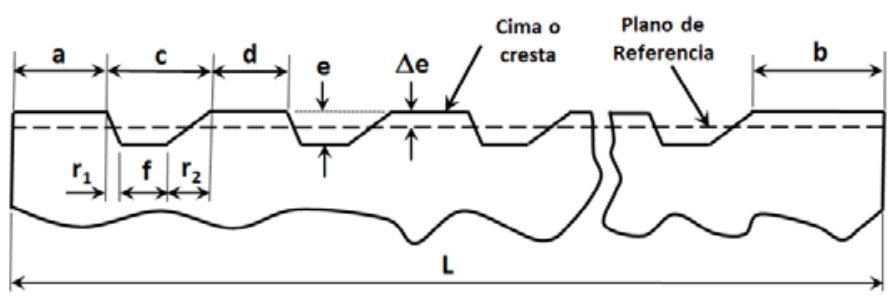

Figura 2. Tribo-sistema de estudio 
problema de estudio se conservó esta simplificación, al igual que lo han hecho otros investigadores (Ronen et al., 2001).

Con base en los supuestos anteriores y asumiendo que la presión sólo permanece constante a lo largo del espesor de la película lubricante, debido a sus pequeñas dimensiones, se han derivado expresiones para la carga sustentada por unidad de longitud $P$, el flujo volumétrico por unidad de tiempo $Q$, la fuerza de fricción por unidad de longitud $F_{f} \mathrm{y}$ el coeficiente de fricción promedio $f_{F f-P R O M}$ en función de $\mu, U$ y la geometría de la cuña convergente (esto es: el ángulo de convergencia de la cuña $\theta$, los espesores máximo y mínimo de la película fluida $h_{1}$ y $h_{2}$ y la longitud del cojinete $l$. Para el caso $\operatorname{del} f_{F f}$ se obtuvo una expresión de la forma,

$f_{F f}=\frac{F_{f}}{P}:=g\left(\frac{\mu U}{P}, \frac{p_{o} l}{P}, \frac{\Delta p l}{P}, \frac{h_{2}}{l}, \alpha,\right)$

donde

$\alpha=h_{1} / h_{2}$ es el parámetro de la película lubricante,

$p_{o} \quad$ es la presión manométrica del lubricante al ingresar a la región de interacción y

$\Delta p$ representa el cambio en la presión del lubricante entre la zona de salida y la de ingreso.

Si $p_{o}=0$ y $\Delta p=0$, la expresión 2 se reduce a

$f_{F f}=(\alpha-1) \cdot\left[\frac{1-\alpha+\frac{2}{3}(\alpha+1) \ln \alpha}{2-2 \alpha+(\alpha+1) \ln \alpha}\right] \cdot\left(\frac{h_{2}}{l}\right)$

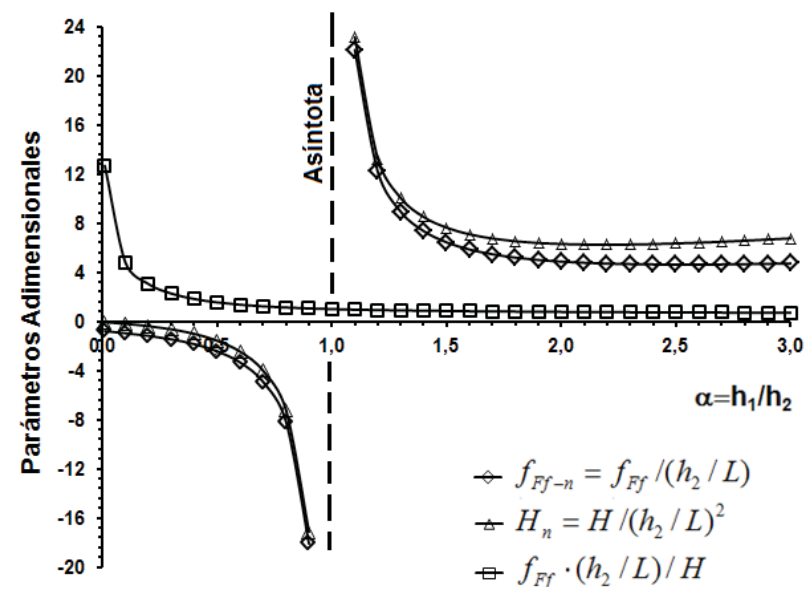

Figura 3. Relaciones entre $f_{F f} \cdot\left(h_{2} / L\right) / H, H_{n}=H /\left(h_{2} / L\right)^{2}$, $f_{F f-n}=f_{F f} /\left(h_{2} / L\right)$ y $\alpha$ de donde se deduce que, con base en los supuestos hechos en el desarrollo de este modelo, se convierte en un coeficiente puramente "geométrico".

En la figura 3 se muestran diversas relaciones entre grupos adimensionales conformados por $f_{F f}, H=\mu \mathrm{U} / \mathrm{P}$, $h_{2} / l$ y $\alpha$.

Los valores de $0<\alpha<1$ hacen referencia a sistemas en los que el flujo a lo largo de las superficies es divergente; mientras que, los valores de $\alpha>1$ se asocian a flujos convergentes.

Esto se conoce como efectos de cuña divergente y convergente, respectivamente. Al mantener fija la relación $h_{2} / l, f_{F f}$ y $H$ se minimizan para $\alpha=2,55$ y $\alpha=2,18$, respectivamente. La figura 3 es una forma condensada de mostrar las soluciones obtenidas al problema de lubricación de Reynolds para un cojinete de longitud infinita (Suárez, 2008).

\section{Análisis dimensional}

Considerando el sistema de estudio propuesto (figura 2), las variables involucradas en el problema a resolver pueden clasificarse en tres grupos, a saber:

- Variables asociadas con la superficie texturizada: $n$ (número de surcos), $L, c, f, e$,

- Variables asociadas a la geometría de la macrocuña convergente: $\theta, h_{2}, L_{r}$,

- Variables asociadas al fluido y/o a las condiciones de operación: $\mu, U, P$.

Es importante notar que de estas variables, $h_{2}$ es una variable que merece consideración especial. $h_{2}$ es una variable derivada, calculable con el modelo propuesto para un cojinete no texturizado; además, puede medirse experimentalmente (Ronen et al., 2001; Etsion et al., 2004). $h_{2}$ es una de las variables más relevantes en la evaluación de la estabilidad de la película lubricante cuando se le relaciona con la rugosidad conjunta del sistema $R_{q}^{*}=\left(R_{q 1}+R_{q 2}\right)^{1 / 2}$ (Hutchings, 1992). También se le puede relacionar con $P$ y $f_{F f}$.

Al efectuar un análisis dimensional sobre el conjunto de variables constituido por los tres grupos de variables antes identificados y el coeficiente de fricción $f_{F f}$ usando el Teorema $\Pi$ de Buckingham (Streeter y Wylie, 1996) se obtuvo una relación de la forma,

$f_{F f}=g_{2}\left(H, D, \beta, \frac{e}{c}, \theta, n\right)$

donde

$H=\mu U / P$ es el parámetro de lubricación, 
$D=n c / L$ es la densidad lineal del texturizado,

$\beta=f / c$ es un parámetro relativo al aspecto de los surcos,

$D$ y $\beta$ al igual que $H$, son grupos adimensionales.

Es importante tener presente que los parámetros de rugosidad superficial relacionados con las alturas de las asperezas normalizados, con respecto a la profundidad de los $\operatorname{surcos} e$ (por ejemplo: la rugosidad media aritmética $R_{a}^{N}$ y la rugosidad media cuadrática $R_{q}^{N}$ normalizadas) y la localización de la línea media para estimarlos $(\Delta e / e)$ dependen específicamente de los grupos $D$ y $\beta$, y por lo tanto, dichos grupos podrían incorporarse a futuro en un modelo más robusto que considere sus efectos en el desempeño a fricción de un cojinete. Las relaciones establecidas para estos parámetros de la micro-topografía superficial en función de los grupos $D$ y $\beta$ son de la siguiente forma,

$\frac{\Delta e}{e}=\left(\frac{1+\beta}{2}\right) D$

$R_{a}^{N}=D\left\{\left(\frac{1-D}{D}\right)\left(\frac{\Delta e}{e}\right)+\beta\left(1-\frac{\Delta e}{e}\right)+\frac{(1-\beta)}{2}\left[1-2\left(\frac{\Delta e}{e}\right)+2\left(\frac{\Delta e}{e}\right)^{2}\right]\right\}$

$R_{q}^{N}=\left\langle D \cdot\left\{\frac{(1-D)}{2}(1+\beta)\left(\frac{\Delta e}{e}\right)+\beta\left(1-\frac{\Delta e}{e}\right)^{2}+(1-\beta)\left[\frac{1}{3}-\left(\frac{\Delta e}{e}\right)+\left(\frac{\Delta e}{e}\right)^{2}\right]\right\}\right\}^{1 / 2}$

para $D \in[0 ; 1]$ y $\beta \in[1 ; 1]$.

Los resultados de este análisis dimensional realizado ayudarán a guiar exploraciones futuras con diversas texturas tendientes a encontrar aquellas que permitan obtener mayores reducciones en las fuerzas de fricción e incrementos más significativos en la capacidad de soporte de carga de cojinetes.

\section{Modelo acoplado para un cojinete texturizado}

En la figura 4 se muestra un cojinete texturizado formado por una serie de $n$ pequeños cojinetes planos. Las variables a encontrar en este modelo son el espesor mínimo de película lubricante $h_{N}$, el caudal volumétrico $Q$, las presiones a la entrada y la salida de cada microcojinete $p_{i}$, las contribuciones a la carga de sustentación a nivel de cada micro-cojinete $P n_{i^{\prime}}$ las contribuciones a la fuerza de fricción total del sistema $F f_{i}$, la fuerza de fricción total del sistema $F f$ y los coeficientes de fricción locales $f_{F f-L O C A L}$ y promedio $f_{F f-P R O M}$.
El conjunto de variables de entrada lo constituyen la geometría del elemento texturizado $\left(L_{r}, n, a, b, c, d, f, r_{1}\right.$, $e$, figura $2 \mathrm{~b}$ ), el ángulo de inclinación de la macro-cuña convergente $(\theta)$ y las condiciones de operación $(U, P, \mu$, $\left.p_{o}=p_{1}, p_{s}=p_{n+1}\right)$.

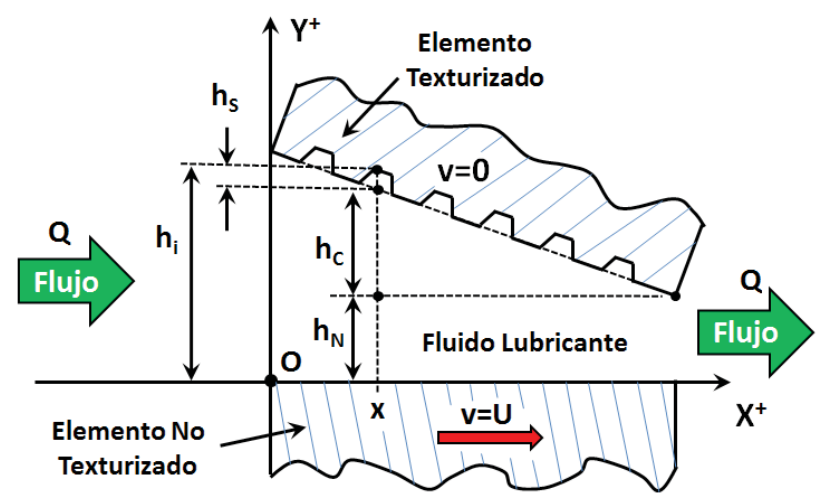

Figura 4. Esquema del cojinete texturizado para la construcción del modelo

En la figura se observa que en las zonas límites entre cada uno de estos microcojinetes, el espesor de la película lubricante puede descomponerse en tres términos,

$h_{i}=h_{\mathrm{N}}+h_{\mathrm{C}}+h_{\mathrm{S}}=h_{\mathrm{N}}+K_{i}$

para $i=1,2, \ldots, n+1$

y donde

$h_{N}=h_{n+1}$ espesor mínimo de la película lubricante a la salida del fluido del cojinete,

$h_{C}=\operatorname{Tan} \theta \cdot\left(L_{p}-x_{i}\right)$ componente variable del espesor de la película relacionada con macro-cuña convergente,

$h_{s} \quad$ componente del espesor de la película relacionada con las desviaciones generadas por el texturizado. Para el caso de estudio, ésta puede ser: $h_{S}=0$, si no se está en el fondo del surco; o $h_{S}=e / \operatorname{Cos} \theta$, si corresponde al fondo del surco.

$K_{i} \quad$ es un coeficiente asociado con la geometría del cojinete, el cual consolida la parte constante y conocida de la geometría de la película lubricante, la cual ante diversas cargas aplicadas experimenta cambios solamente en $h_{N}$.

Aplicando el principio de conservación de la masa para un fluido lubricante incompresible, se establecieron las siguientes ecuaciones en cada micro-cojinete,

$Q=Q_{i}=U C_{i}-\Delta p \cdot D_{i} \quad$ para $i=1, \ldots, n$ 
donde

$C_{i}=\frac{h_{i} \cdot h_{i+1}}{h_{i}+h_{i+1}}$

$D_{i}=\frac{1}{6 \mu \cdot l_{i}} \cdot \frac{\left(h_{i} \cdot h_{i+1}\right)^{2}}{\left(h_{i}+h_{i+1}\right)}$

Usando el modelo de Reynolds, puede mostrarse que la carga por unidad de longitud de un cojinete con un texturizado como el esquematizado en la figura 4 está dada por la siguiente expresión,

$$
\begin{aligned}
P=\sum_{i=1}^{n} p_{i} l_{i} & +\sum_{i=1}^{n}\left[l_{i}\left(p_{i+1}-p_{i}\right) \cdot\left(\frac{h_{i+1}}{h_{i}+h_{i+1}}\right)\right]+6 \mu U \\
& \left\{\frac{\operatorname{Lnh}_{1}}{\operatorname{Tan}^{2} \theta_{1}}-\frac{\operatorname{Lnh}_{n+1}}{\operatorname{Tan}^{2} \theta_{n}}+2 \sum_{i=1}^{n}\left[\frac{1}{\operatorname{Tan}^{2} \theta_{i}} \cdot\left(\frac{\Delta h_{i}}{h_{i}+h_{i+1}}\right)\right]\right. \\
& \left.+\sum_{i=2}^{n}\left[\operatorname{Lnh}_{1} \cdot\left(\frac{1}{\operatorname{Tan}^{2} \theta_{i}}-\frac{1}{\operatorname{Tan}^{2} \theta_{i-1}}\right)\right]\right\}
\end{aligned}
$$

La ecuación 9 puede resolverse empleando un método iterativo en el cual se conocen $Q, p_{o}$ y $p_{s} ;$ allí, la ecuación 10 controla el proceso iterativo al utilizarse para evaluar el error admisible pre-establecido en el cálculo de la carga, con respecto a su valor nominal $P$; debe considerarse que el espesor mínimo de película lubricante disminuye con el incremento de la carga normal.

a) Relación entre $P$ y e

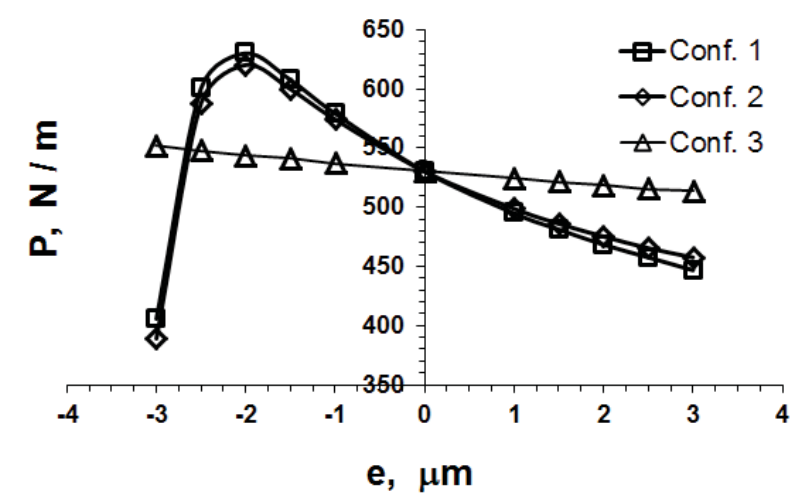

Una vez determinados $h_{N}, Q$, y $p_{i}$, se pueden calcular las contribuciones de carga normal y tangenciales (componentes de la fuerza de fricción) asociadas a cada micro-cojinete. Con la suma de éstas últimas se determina la fuerza de fricción total. Finalmente, los coeficientes de fricción se determinan mediante las siguientes relaciones,

$f_{F f-P R O M}=E f / P$

$f_{\text {Ff-LOCAL-i }}=E f_{i} / P n_{i}$

\section{Implementación de resultados}

El modelo se implementó en el programa denominado EXPLOTEX-MC4E (Explorador de Texturizados) mediante el uso de MatLab 6.1 (Suárez, 2008). Los resultados iniciales obtenidos en una pequeña exploración inicial realizada con la herramienta desarrollada se muestran en la figura 5. En ella se observa cómo varían $P$ y $f_{\text {Ff-PROM }}$, con respecto a la profundidad de los "sur$\cos ^{\prime \prime} e$, para tres configuraciones micro-topográfícas, cuyas características se detallan en la tabla adjunta a esta figura y están basadas en el esquema mostrado en la figura $2 \mathrm{~b}$. Considerando la convención tomada en el modelo propuesto, se deduce que los valores negativos de $e$ indican un cambio en la micro-topografía general tomada como punto de partida, esto es, donde se suponen mesetas en realidad existen depresiones y viceversa. Como puede observarse, existen arreglos micro-topográficos que simultáneamente, maximizan la carga sustentada y minimizan el coeficiente de fricción.

b) Relación entre $f_{\text {Ff-PROM }}$ y e

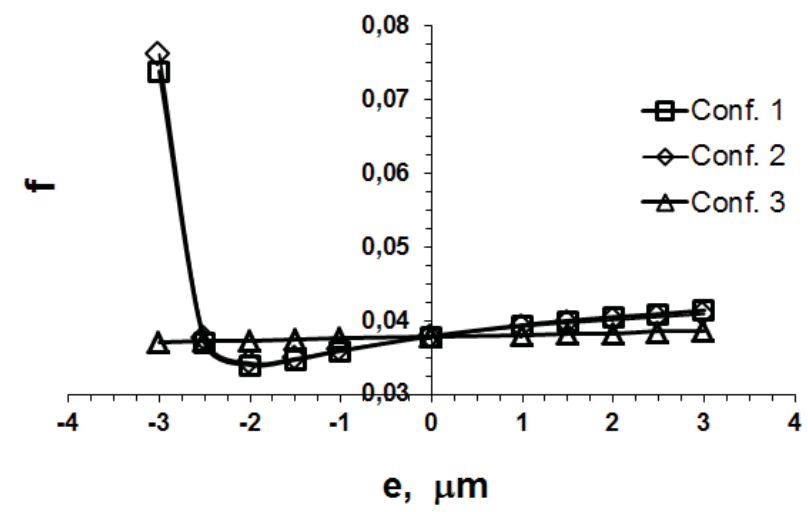

Figura 5. Capacidad de soporte de carga y coeficiente de fricción en función de la profundidad de los surcos para tres configuraciones micro-topográficas 
Los valores empleados en la simulación para las variables tomadas como fijas fueron: $n=50, L=25 m m, \theta=3^{\circ}$, $U=1 \mathrm{~m} / \mathrm{s}, v=46 \cdot 10^{-6} \mathrm{~m}^{2} / \mathrm{s}, \rho_{\text {Lubricante }}=900 \mathrm{~kg} / \mathrm{m}^{3}$ y $a=0$ (figura 2). En cada curva, cada punto representa una textura en particular (un cojinete distinto); cuando $e=0$, las distintas configuraciones propuestas confluyen a la condición de no textura y representan a un mismo cojinete, un cojinete plano y liso, que para el caso simulado tiene una macro-cuña de $\theta=3^{\circ}$. El efecto de las texturas (y específicamente de las micro-cuñas cuya formación promueven) puede medirse al considerar la diferencia entre la carga sustentada o el coeficiente de fricción asociados a una textura en particular, con respecto a la condición de no textura (la cual sólo considera el efecto de la macro-cuña). En los casos presentados en la figura 5, para valores de $e=-2 \mu \mathrm{m}$, las configuraciones 1 y 2 permiten incrementar la capacidad de soporte de carga en un $17 \%$ y reducir el coeficiente promedio de fricción en un $10 \%$, con respecto a la condición de no textura.

Tabla. Configuraciones micro-topográficas

\begin{tabular}{lccc}
\hline \multicolumn{1}{c}{ Variable } & Conf. 1 & Conf. 2 & Conf. 3 \\
\hline$b=d, \mu m$ & 425 & 150 & 25 \\
$c, \mu m$ & 75 & 350 & 475 \\
$f, \mu m$ & 25 & 25 & 150 \\
$r_{1}, \mu m$ & 25 & 300 & 25 \\
\hline
\end{tabular}

Los resultados de esta pequeña exploración del modelo indican que efectivamente la textura superficial de un cojinete induce cambios significativos en su desempeño (figura 5). Además, pone de manifiesto que no necesariamente una superficie más lisa (o menos rugosa si se prefiere) es la que permite alcanzar coeficientes de fricción más bajos y mayores capacidades de soporte de carga. Sin embargo, sólo algunas superficies rugosas permiten mejorar la capacidad de soporte de carga y reducir el coeficiente de fricción promedio del sistema. En el caso de las configuraciones 1 y 2, estas mejoras sólo son posibles para valores de $e \in(-3 ; 0)$ aproximadamente, considerando el significado de $e<$ 0 según las convenciones empleadas en el desarrollo del modelo. Texturas asociadas a estas dos configuraciones con valores de $e$ fuera de este rango, incrementan la fricción y reducen la capacidad de carga del sistema. De otro lado, esto no debe considerarse a la ligera como algo negativo, pues es posible tener aplicaciones donde por ejemplo se requiera incrementar la fricción. De esta manera, el texturizado de superficies es una herramienta útil para controlar la fricción y el desempeño de un tribosistema como el estudiado en este trabajo.

Un análisis más detallado de los efectos individuales y combinados de los diferentes parámetros que definen la textura estudiada en este artículo (empleando el modelo construido), permitirá explicar por qué existen configuraciones diferentes con comportamientos muy similares (configuraciones 1 y 2 por ejemplo) y por qué en algunas configuraciones las variaciones de $e$ sólo inducen cambios ligeros y graduales en su comportamiento (configuración 3) mientras que en otras, dichos cambios son más abruptos (configuraciones 1 y 2). Estas y otras preguntas por resolver, serán los elementos que guíen una exploración más robusta sobre el efecto de la texturas acondicionadas en el desempeño tribológico de cojinetes planos operando en condiciones HL, la cual podrá efectuarse eficientemente con la ayuda del modelo presentado en este trabajo.

\section{Conclusiones}

Con respecto al trabajo presentado en este artículo se concluye que:

- El modelo numérico propuesto (condensado las ecuaciones 8 a 12) permite establecer relaciones entre las condiciones de operación (carga, velocidad y viscosidad dinámica), el espesor mínimo de película lubricante, el coeficiente de fricción y la micro-topografía acondicionada de un cojinete plano de deslizamiento operando en régimen hidrodinámico (HL) para un cojinete cuya textura acondicionada está constituida por surcos trapezoidales orientados perpendicularmente con respecto a la dirección de deslizamiento.

- El modelo permite realizar exploraciones rápidas en sistemas de cojinetes planos de deslizamiento y a diferencia de otros modelos de la literatura, posibilita evaluar el efecto combinado de las micro-cuñas que operan como micro-cojinetes, y la macro-cuña convergente típica de estos elementos de estudio. También permite cuantificar los aportes individuales de la macro-cuña convergente y del texturizado como tal para condiciones de operación específicas (carga, velocidad y viscosidad dinámica).

- Los resultados obtenidos con la pequeña exploración efectuada en un conjunto reducido de texturas indican que efectivamente la textura superficial o la microtopografía de las asperezas juega un papel relevante en el desempeño tribológico de un cojinete plano de deslizamiento. 
Como trabajo futuro a desarrollar con el modelo, está la evaluación de un conjunto mayor de texturas con el fin de hacer una exploración más amplia, sistemática y detallada.

Para eso se sugiere hacer uso de algunos de los grupos adimensionales hallados mediante análisis dimensional (principalmente $f_{\text {Fricción }}=g(\mu U / P, D, \beta$, $e / c)$ ) con el fin de guiar y simplificar el diseño de experimentos virtuales. De esta manera, se podrá caracterizar mejor la sensibilidad del modelo y evaluar su rango de aplicación según el mecanismo sobre el cual éste se sustenta, la presencia de micro-cojinetes en la escala de la rugosidad.

\section{Agradecimientos}

Los autores expresan sus agradecimientos a la Facultad de Minas de la Universidad Nacional de Colombia, Sede Medellín por los recursos brindados para la realización de este trabajo.

\section{Referencias}

Anno J.N., Walowit J.A., Allen C.M. Microasperity Lubrication. Journal of Lubrication Technology, abril 1968: 351-357.

Bowden F.P., Tabor D. The Friction and Lubrication of Solids, Oxford, Clarendon Press, 2001, 253 p.

Chiang H.L., Chou T.L., Hsu Ch.H., Hsu C.H., Lin J.R. Surface Roughness Effects on Dynamic Characteristics of Finite Slider Bearings. Journal of C.C.I.T., volumen 34 (número 1), noviembre 2005: 1-11.

Christensen H. Sochastic Models for Hydrodynamic Lubrication of Rough Surfaces. Proc Instn Mech Engrs, volumen 184 (número 55) Pt1, 1969-1970: 1013-1022.

Christensen H., Tonder K. The Hydrodynamic Lubrication of Rough Bearing Surfaces of Finite Width. Transctions of ASME, julio 1971: 324-330.

Erdemir A. Review of Engineered Tribological Interfaces for Improved Boundary Lubrication. Tribology International, volumen 38 (número 3), 2005: 249-256.

Etsion I., Halperin G., Brizmer V., Kligerman Y. Experimental Investigation of Laser Surface Textured Parallel thrust Bearings. Tribology Letters, volumen 17 (número 2), agosto 2004: 295-300

Hutchings I.M. Tribology: Friction and Wear of Engineering Materials, Londres, Edward Arnold, 1992, pp. 58-76.

Kligerman Y., Etsion I. Experimental Investigation of Laser Surface Texturing for Reciprocating Automotive Components. Tribology Transactions, volumen 45 (número 4), 2002: 444-449.
Kovalchenko A., Ajayi O., Erdemir A., Fenske G., Etsion I. The Effect of Laser Surface Texturing on Transitions in Lubrication Regimes During Unidirectional Sliding Contact. Tribology International (número 38), 2005: 219-225.

Letalleur N., Plouraboué F., Prat M. Écoulement de lubrifiant entre surfaces rugueuses en mouvement relatif, Institut de Mécanique des Fluides de Toulouse. La Revue de Métallurgie - CIT/ Science et Gene des Matériaux, mayo 2001, pp. 455-458.

Pettersson, U., Jacobson, S. Influence of Surface Texture on Boundary Lubricated Sliding Contacts. Tribology International (número 36), 2003: 857-864.

Pettersson U. Surfaces Designed for High and Low Friction, tesis (doctorado en filosofía), Uppsala Universitet, 2005, 44 p.

Ronen A., Etsion I., Kligerman Y. Friction-Reducing Surface-Texturing in Reciprocating Automotive Components. Tribology Transactions, volumen 44 (número 3), 2001: 359-366.

Ryk G., Kligerman Y., Etsion I. Experimental Investigation of LASER Surface Texturing for Reciprocating Automotive Components. Tribology Transactions, volumen 45 (número 4), 2002: 444-449.

Streeter V., Wylie E.B. Fluid Mechanics, 8a ed., USA, McGraw Hill, 1996, $594 \mathrm{p}$.

Suarez F.A. Efecto de la textura superficial en el desempeño a fricción de un cojinete plano de deslizamiento, tesis (maestría), Medellín, Colombia, Universidad Nacional de Colombia, 2008, 124 p.

Schlichting H. Boundary Layer Theory, USA, McGaw-Hill, 1979, pp. 116-123.

Wang Q.J., Zhu D. Virtual Texturing: Modelling the Performance of Lubricated Contacts of Engineered Surfaces. Transactions of the ASME, volumen 127, octubre 2005: 722-728.

Tonder K. Hydrodynamic Effects of Tailored Inlet Roughness Extended Theory. Tribology International, volumen 37 (número 2), 2004: 137-142.

Wang X., Adachi K., Otsuka K., Kato K. Optimization of the Surface Texture for SiC Sliding in Water (artículo en prensa), Applied Surface Science, 2006.

Wang X., Kato K., Adachi K., Aizawa K. Loads Carrying Capacity Map for the Surface Texture Design of SiC Thrust Bearing Sliding in Water. Tribology International, volumen 36 (número 3), 2003: 189-197.

Wang Q.J., Zhu D. Virtual Texturing: Modeling the Performance of Lubricated Contacts of Engineered Surfaces. Transactions of the ASME, volumen 127, octubre 2005: 722-728. 


\section{Semblanza de los autores}

Fabio Alexander Suárez-Bustamante. Realizó sus estudios de ingeniería mecánica en la Facultad de Minas de la Universidad Nacional de Colombia (UN), donde obtuvo grado de honor por su trabajo en el área de tribología sobre el análisis del modelo de desgaste propuesto por Archard. Realizó su maestría en la misma universidad graduándose en el año 2008. Sus principales temas de interés incluyen desgaste por deslizamiento en seco, diseño de superficies de ingeniería, lubricación hidrodinámica y mecánica del contacto. Actualmente dicta cursos de tratamientos térmicos a materiales metálicos y propiedades mecánicas en la UN y es miembro activo del Grupo de Tribología y Superficies y del Grupo de Tecnología de Materiales de esta universidad.

Juan Manuel Vélez-Restrepo. Ingeniero mecánico por la Universidad Nacional de Colombia (Facultad de Minas, Medellín). Realizó sus estudios de maestría y doctorado (1997) en la Universidad de Sao Pablo, Brazil. Desde 1985 es profesor de la Universidad Nacional de Colombia (UN), donde ha dictado cursos de ciencia de materiales e ingeniería, transformaciones de fases y tribología entre otros. Ha sido director del Departamento de Ingeniería de Materiales (2003); director de investigación de la Facultad de Minas (2002-2003), decano de la misma en el periodo 2006-2010. Coordinador del grupo de investigación en ciencia y tecnología de materiales y director del Proyecto Leonardo da Vinci, que promueve la alfabetización científica y tecnológica y la innovación en el aprendizaje.

Francisco Mauricio Toro-Botero. Estudió ingeniería civil en la Facultad de Minas de la Universidad Nacional de Colombia (UN). Realizó sus estudios de maestría y doctorado en la Universidad de Mississipi (EU) en el área de hidro-ciencias e ingeniería. Durante el desarrollo de su doctorado se destacó como el mejor estudiante de la Facultad de Ingeniería (Universidad de Mississippi, 1992). Desde 1986 se desempeña como profesor asociado de la Universidad Nacional de Colombia (Sede Medellín, Facultad de Minas) donde ha dictado numerosos cursos en pregrado y posgrado, relacionados con manejo de calidad de aguas (modelamiento), turbulencia, hidráulica transitoria, simulación hidrodinámica, hidráulica fluvial y métodos numéricos entre otros.

\section{Este artículo se cita:}

\section{Chicago citation}

Suárez-Bustamante, Fabio Alexander, Francisco Mauricio Toro-Botero, Juan Manuel Vélez-Restrepo. Influencia de texturas superficiales acondicionadas en el desempeño de cojinetes planos operando en condiciones HL. Ingeniería Investigación y Tecnología, XIII, 03 (2012): $\mathrm{xxx}-\mathrm{xxx}$.

\section{Citación ISO 690}

Suárez-Bustamante F.A., Toro-Botero F.M., Vélez-Restrepo J.M. Influencia de texturas superficiales acondicionadas en el desempeño de cojinetes planos operando en condiciones HL. Ingeniería Investigación y Tecnología, volumen XIII (número 3), julio-septiembre 2012: xxx-xxx. 